\title{
Effective Cahn-Hilliard equation for the phase separation of active Brownian particles
}

\author{
Thomas Speck, ${ }^{1}$ Julian Bialké, ${ }^{2}$ Andreas M. Menzel, ${ }^{2}$ and Hartmut Löwen ${ }^{2}$ \\ ${ }^{1}$ Institut für Physik, Johannes Gutenberg-Universität Mainz, Staudingerweg 7-9, 55128 Mainz, Germany \\ ${ }^{2}$ Institut für Theoretische Physik II, Heinrich-Heine-Universität, D-40225 Düsseldorf, Germany
}

The kinetic separation of repulsive active Brownian particles into a dense and a dilute phase is analyzed using a systematic coarse-graining strategy. We derive an effective Cahn-Hilliard equation on large length and time scales, which implies that the separation process can be mapped onto that of passive particles. A lower density threshold for clustering is found, and using our approach we demonstrate that clustering first proceeds via a hysteretic nucleation scenario and above a higher threshold changes into a spinodal-like instability. Our results are in agreement with particle-resolved computer simulations and can be verified in experiments of artificial or biological microswimmers.

PACS numbers: 82.70.Dd,64.60.Cn

The collective behavior of living "active" matter has recently attracted considerable interest from the statistical physics community (for reviews, see Refs. 1, 2). Even if the mutual interactions of the individual units are following simple rules, complex spatiotemporal patterns can emerge. Examples in nature occur on a wide range of scales from flocks of birds 3 to bacterial turbulence [4]. A basic physical model is obtained by describing the individual entities as particles with internal degrees of freedom (in the simplest case just an orientation) that consume energy and are thus driven out of thermal equilibrium. Consequently, shaken granular particles [5] and phoretically propelled colloidal particles $[6]$ have been investigated in detail. Moreover, the observed collective behavior might find applications in, e.g., the sorting [10] and transport of cargo [11.

Here we are interested in the phase behavior of repulsive particles below the freezing density. While in equilibrium only one fluid phase exists, sufficiently dense suspensions of repulsive self-propelled disks undergo an "active phase separation", i.e., particles aggregate into a dense, transiently ordered cluster surrounded by a dilute gas phase. This has been observed first [12, 13, in computer simulations of a minimal model [14 16. Clustering has also been reported in experiments using colloidal suspensions of active Brownian particles, in which the particles are phoretically propelled along their orientations due to the catalytic decomposition of hydrogen peroxide on a platinum hemisphere [7, or due to lightactivated hematite [8]. In these experiments, phoretic attractive forces play an important role. A closer realization of ideally repulsive particles is possible through the reversible local demixing of a near-critical water-lutidine mixture [17. Colloidal particles propelled due to the ensuing local density gradients show indeed the predicted phase separation [9]. While in passive suspensions phaseseparation occurs only for sufficiently strong attractive forces, the microscopic mechanism for repulsive active particles is due to self-trapping: colliding particles block each other due to the persistence of their orientation [9]. In sufficiently dense suspensions, the "pressure" of the free, fast particles leads to the growth of small clusters until phase separation is reached. This generic dynamical instability due to a density-dependent mobility has been first studied by Tailleur and Cates for the run-and-tumble motion of bacteria [18] and later also for active Brownian particles [19]. At even higher densities, first steps have been taken to study glassy dynamics [20, 21] and crystallization 22, 23. Another interesting question is the interplay of the propulsion with attractive forces $24-26$.

While the phase separation and nucleation in passive suspensions has been studied extensively, an open fundamental question is whether the clustering of active Brownian particles, which is an intrinsically nonequilibrium system, can be mapped on the phase separation dynamics of passive particles. In this Letter, we demonstrate for a simple model system of Brownian particles that this mapping exists on coarse-grained length and time scales. We find a formal analogy with the Cahn-Hilliard equation, which indeed implies a mapping of the phase separation of active Brownian particles to the phase separation process of passive particles governed by attractive forces. This allows to translate established concepts to the study of active systems and, moreover, implies that statistical properties (growth exponent, scaling of clusters, etc.) are unchanged in active systems. We comment that recently additional terms in the dynamics of phase separation have been studied [16, 27, which are not derivable from an effective free energy. Our crucial point here is that simple, genuinely active systems do not generally need these non-Hamiltonian terms in order to be described correctly. Moreover, we predict by a weakly nonlinear stability analysis - and confirm our prediction through particle-resolved computer simulations - that the nature of the separation process (spinodal decomposition or hysteretic nucleation-like behavior) depends on the propulsion speed along the instability line. While it corresponds to a spinodal decomposition for small speeds (high density), it changes to a hysteretic nucleation-like behavior upon crossing a threshold. The actual instability line predicted by our analysis is in good agreement with the simulation data. 
The minimal model for active Brownian particles 12 16] that we study consists of $N$ repulsive disks in two dimensions, the motion of which is governed by

$$
\dot{\mathbf{r}}_{k}=-\nabla U+v_{0} \mathbf{e}_{k}+\boldsymbol{\eta}_{k} .
$$

Particles interact via the potential energy $U$, and $\boldsymbol{\eta}_{k}$ is the Gaussian white noise describing the influence of the solvent. In addition, particles are propelled with constant speed $v_{0}$ along their orientations $\mathbf{e}_{k}$, which undergo free rotational diffusion with diffusion coefficient $D_{\mathrm{r}}$ and are, therefore, uncorrelated. In an effort to connect these microscopic equations of motion with the emerging largescale behavior of the suspension, we have recently derived the effective hydrodynamic equations

$$
\begin{gathered}
\partial_{t} \rho=-\nabla \cdot[v(\rho) \mathbf{p}-D \nabla \rho], \\
\partial_{t} \mathbf{p}=-\frac{1}{2} \nabla[v(\rho) \rho]+D \nabla^{2} \mathbf{p}-D_{\mathrm{r}} \mathbf{p}
\end{gathered}
$$

starting from the full $N$-body Smoluchowski equation for the evolution of the joint probability distribution of all particle positions and their orientations [15. Here, $D$ denotes the long-time diffusion coefficient of the passive suspension. Eqs. (2) and (3) have been derived under the assumption of spatially slowly varying number density $\rho(\mathbf{r}, t)$ and orientational field $\mathbf{p}(\mathbf{r}, t)$. Instead of assuming a phenomenological functional form for the effective speed $v(\rho)$ (see Refs. [12, 28]), we have shown that for the minimal model close to the instability line the linear relation $v(\rho)=v_{0}-\rho \zeta$ follows, where $\zeta$ quantifies the force imbalance due to the self-trapping.

Before we continue, we simplify the equations through choosing $1 / D_{\mathrm{r}}$ as the unit of time, $\sqrt{D / D_{\mathrm{r}}}$ as the unit of length, and we normalize both fields by the average density, $\rho \mapsto \bar{\rho}(1+\delta \rho)$ and $\mathbf{p} \mapsto \bar{\rho} \mathbf{p}$. The equations then read

$$
\begin{gathered}
\partial_{t} \delta \rho=-\alpha \nabla \cdot \mathbf{p}+\nabla^{2} \delta \rho+4 \xi \nabla \cdot(\mathbf{p} \delta \rho), \\
\partial_{t} \mathbf{p}=-\beta \nabla \delta \rho+\nabla^{2} \mathbf{p}-\mathbf{p}+4 \xi \delta \rho \nabla \delta \rho,
\end{gathered}
$$

where we have separated the non-linear terms. The dimensionless coefficients appearing here are defined as

$$
\xi \equiv \frac{\bar{\rho} \zeta}{v_{*}}, \quad \alpha \equiv 4\left(v_{0} / v_{*}-\xi\right), \quad \beta \equiv 2\left(v_{0} / v_{*}-2 \xi\right)
$$

with characteristic speed $v_{*} \equiv 4 \sqrt{D D_{\mathrm{r}}}$.

Dropping the non-linear terms in Eqs. (4) and (5), it is straightforward to investigate the linear stability of the homogeneous solution $\delta \rho=0$ and $\mathbf{p}=0$. Indeed, depending on the values of the coefficients $\alpha$ and $\beta$, the homogeneous density profile might become instable. The dispersion relation

$$
\sigma(q)=-\frac{1}{2}-q^{2}+\frac{1}{2} \sqrt{1-4 \alpha \beta q^{2}} \approx-(1+\alpha \beta) q^{2}
$$

quantifies the growth rate of a perturbation with wave vector $q$. On large scales (small $q$ ), the instability occurs whenever $1+\alpha \beta<0$. From the condition $1+\alpha \beta=0$, we determine the value of the dimensionless force imbalance coefficient

$$
\xi_{\mathrm{c}}=\frac{3}{4}\left(v_{\mathrm{c}} / v_{*}\right)-\frac{1}{4} \sqrt{\left(v_{\mathrm{c}} / v_{*}\right)^{2}-1}
$$

at the onset of the instability for a given critical speed $v_{\mathrm{c}}$ [15. Clearly, $v_{*}$ is the smallest propulsion speed for which the instability is possible, $v_{\mathrm{c}} \geqslant v_{*}$.

In the linear analysis, a small initial perturbation grows unbounded. Of course, due to the non-linear terms implying a coupling to other modes, the amplitude of the perturbation will saturate. We now aim to derive an equation of motion that describes the evolution of an initial perturbation for propulsion speeds $v_{0}=v_{\mathrm{c}}(1+\varepsilon)$ in the vicinity of the linear stability limit 29. The fastest growing wave vector following Eq. (7) is $q_{\mathrm{c}}=\frac{1}{2} \sqrt{(\alpha \beta)^{-1}-\alpha \beta}$, which dominates the initial stage of the developing instability. Expanding $\alpha=\alpha_{0}+\varepsilon \alpha_{1}+\cdots$ and $\beta=\beta_{0}+\varepsilon \beta_{1}+\cdots$ we find $q_{\mathrm{c}} \sim \sqrt{\varepsilon}$ with $\alpha_{0} \beta_{0}=-1$. The growth rate of this mode is $\sigma\left(q_{\mathrm{c}}\right) \approx-\varepsilon \sigma_{1} q_{\mathrm{c}}^{2} \sim \varepsilon^{2}$ to lowest order, where we have defined $\sigma_{1} \equiv \alpha_{0} \beta_{1}+\alpha_{1} \beta_{0}$.

We are interested in the large-scale behavior of the suspension. As suggested by the scaling of critical wave vector and growth rate, we rescale length with $1 / \sqrt{\varepsilon}$ and time with $1 / \varepsilon^{2}$, amounting to $\partial_{t} \mapsto \varepsilon^{2} \partial_{t}$ and $\nabla \mapsto \sqrt{\varepsilon} \nabla$. Matching powers suggests to expand

$$
\delta \rho=\varepsilon c+\varepsilon^{2} c^{(2)}+\cdots, \quad \mathbf{p}=\sqrt{\varepsilon}\left[\varepsilon \mathbf{p}^{(1)}+\varepsilon^{2} \mathbf{p}^{(2)}+\cdots\right] .
$$

To lowest order in $\varepsilon$, we find $\mathbf{p}^{(1)}=-\beta_{0} \nabla c$ for the orientational field leading to

$$
0=\left(1+\alpha_{0} \beta_{0}\right) \nabla^{2} c,
$$

which reproduces the result of the linear stability analysis as required. Gathering terms of the next order leads to

$$
\begin{gathered}
\partial_{t} c=-\alpha_{0} \nabla \cdot \mathbf{p}^{(2)}-\alpha_{1} \nabla \cdot \mathbf{p}^{(1)}+\nabla^{2} c^{(2)}+4 \xi_{\mathrm{c}} \nabla \cdot\left[c \mathbf{p}^{(1)}\right], \\
0=-\beta_{0} \nabla c^{(2)}-\beta_{1} \nabla c+\nabla^{2} \mathbf{p}^{(1)}-\mathbf{p}^{(2)}+4 \xi_{c} c \nabla c .
\end{gathered}
$$

Solving the second equation for $\mathbf{p}^{(2)}$ and plugging the result together with $\mathbf{p}^{(1)}$ into the first equation, we first note that the terms containing $c^{(2)}$ drop out. We, therefore, obtain an evolution equation for the large-scale density fluctuations $c(\mathbf{r}, t)$ alone,

$$
\partial_{t} c=\sigma_{1} \nabla^{2} c-\nabla^{4} c-2 g \nabla \cdot(c \nabla c)=\nabla^{2} \frac{\delta F}{\delta c},
$$

which is the central result of this Letter. Here, $g \equiv$ $2 \xi_{\mathrm{c}}\left(\alpha_{0}+\beta_{0}\right) \geqslant 0$ determines the strength of the nonlinear term, where the equal sign holds for the smallest possible critical speed $v_{\mathrm{c}}=v_{*}$.

We recognize Eq. (11) as the celebrated Cahn-Hilliard equation 30] routinely employed to study phase separation dynamics. It implies the existence of an effective 
free energy functional

$$
F[c]=\int \mathrm{d} \mathbf{r}\left[\frac{1}{2}|\nabla c|^{2}+f(c)\right]
$$

with bulk free energy density $f(c)=\frac{1}{2} \sigma_{1} c^{2}-\frac{1}{3} g c^{3}$. Following our analysis, no "active" non-integrable terms enter the interfacial free energy. The expression for $f(c)$ found here misses the customary $c^{4}$ term stabilizing the high density phase at a finite value for the density. Physically, there is an upper bound to $c$ due to the volume exclusion between particles. This is not contained in the effective hydrodynamic description (2) and (3) of a single tagged particle but complementarily considered in the following.

We now want to test to what extent such an effective free energy agrees with particle-resolved simulation data. To this end, we have performed Brownian dynamics simulations of Eq. (1) for $N=4900$ particles. Particles interact pairwisely via the repulsive WCA potential, the parameters of which have been obtained previously by matching experimental data 9]. For the simulations, we fix the particle diameter $a$, the free diffusion coefficient $D_{0}$, and the rotational diffusion coefficient $D_{\mathrm{r}}=3 D_{0} / \mathrm{a}^{2}$. We vary the propulsion speed $v_{0}$ and the area fraction $\phi=N \pi a^{2} /(2 L)^{2}=\left(\pi a^{2} / 4\right) \bar{\rho}$, where $L$ is the edge length of the simulation box employing periodic boundary conditions. We measure the degree of clustering through the average fraction $P$ of particles that are part of the largest cluster, cf. Refs. 9, 15, which is determined from steady state trajectories. We equilibrate the passive suspension at the desired density, turn on $v_{0}$, and let the system relax into the steady state. The phase diagram is presented in Fig. 1(a), where for every simulated state point $\left(\phi, v_{0}\right)$ the order parameter $P$ is shown. We use a simple threshold such that for $P \geqslant 0.1$ we consider the suspension to be in the cluster phase as indicated by a closed symbol. We also measure the bond orientational order to decide whether the suspension has become a solid as indicated by triangles. In qualitative agreement with other simulations [14, 22, the propulsion melts the solid before entering the cluster phase.

In Fig. 1(b), the bulk free energy density $f(c)$ is sketched for speeds $v_{0}>v_{\mathrm{c}}$ slightly above the critical speed. The form of $f(c)$ implies that the homogeneous density profile with $c=0$ becomes unstable. Following the double tangent construction, phase separation into a dense phase with $c_{+}$and a dilute gas phase with $c_{-}$will occur. The corresponding area fractions $\phi_{ \pm}=\phi\left(1+\varepsilon c_{ \pm}\right)$ follow from the expansion Eq. (9). From the simulations, we expect the area fraction $\phi_{+}$of the dense phase to be nearly close-packed.

In order to obtain a more tractable expression, suppose we know a point $\left(\phi_{1}, v_{1}\right)$ on the instability line, see Fig. 1.(c). Increasing the speed to $v_{2}=v_{1}(1+\varepsilon)$, phase separation is predicted to occur with area fraction $\phi_{-}=\phi_{1}\left[1+\varepsilon c_{-}\left(\phi_{1}, v_{1}\right)\right]$ of the gas phase. Hence, with (a)

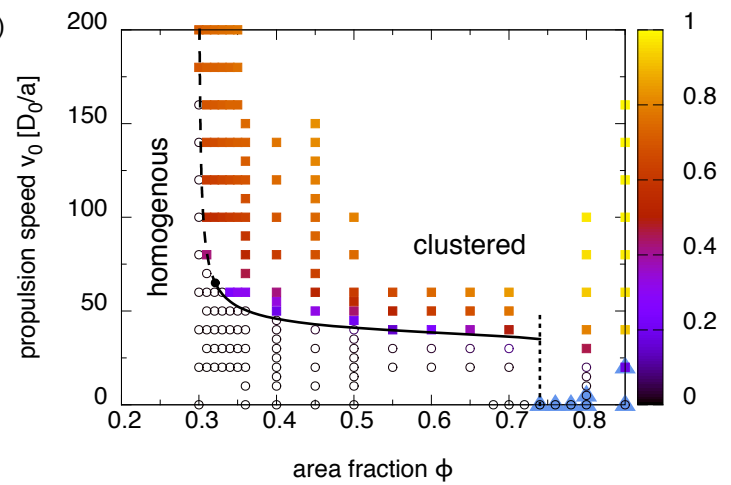

(b)
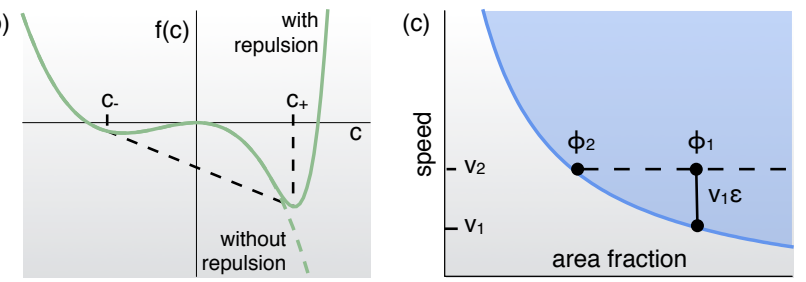

FIG. 1: (a) Instability diagram for repulsive self-propelled disks as a function of area fraction $\phi$ and propulsion speed $v_{0}$. The symbol color indicates the fraction of particles that are part of the largest cluster. The open symbols correspond to the homogeneous suspension $(P<0.1)$ and closed squares to the cluster phase. The vertical dotted line marks the freezing density for the passive $\left(v_{0}=0\right)$ suspension, closed triangles indicate solid. The instability line is calculated from the analytical result Eq. (13). A change from a continuous (solid) to a discontinuous (dashed) transition at the density $\phi_{0} \simeq 0.32$ occurs. (b) Illustration of the double tangent construction. We also sketch the bulk free energy density $f(c)$ for $\sigma_{1}<0$ (dashed line). (c) Derivation of Eq. 13): Given a point $\left(\phi_{1}, v_{1}\right)$ on the instability line, at the slightly larger speed $v_{2}=v_{1}(1+\varepsilon)$ phase separation into the dense phase and the dilute gas phase with area fraction $\phi_{2}=\phi_{-}$will occur.

$\phi_{2}=\phi_{-}$we have found a second point on the instability line. Eliminating $\varepsilon$ and taking the limit $v_{2} \rightarrow v_{1}$ leads to the equation

$$
\frac{\mathrm{d} \phi}{\mathrm{d} v}=\frac{\phi}{v} c_{-}(\phi, v),
$$

which is formally equivalent to the Clausius-Clapeyron equation quantifying the slope along the instability line. However, here the system is intrinsically driven out of equilibrium. We estimate the instability line by numerically solving Eq. (13). To this end, we approximate $c_{-}(\phi, v) \approx \sigma_{1} / g$ by the local minimum of $f(c)$. While we found an analytical expression for $g\left(v_{0} / v_{*}\right)$, the coefficient $\sigma_{1}$ is difficult to estimate from the simulations. As indicated by the numerical phase diagram Fig. 1(a), there is not only a minimal speed $v_{*}$ but also a minimal density $\phi_{*}$ for clustering to occur. Close to this lower density $\sigma_{1}(\phi) \propto \phi_{*}-\phi$ should hold and we use this expression for $\sigma_{1}$ throughout with $\phi_{*}=0.29$ as a fit parameter. To obtain a continuous function $v_{*}(\phi)$, we fit the numeri- 

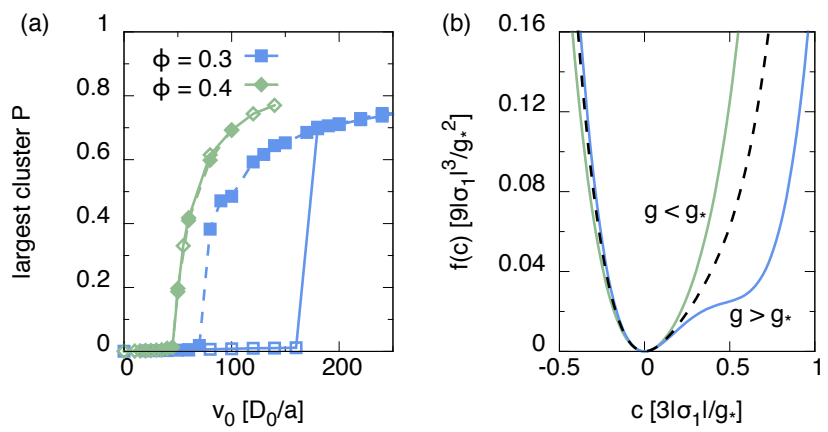

FIG. 2: (a) Hysteresis at low area fraction $\phi$ and vanishing at higher values of $\phi$. Shown is the steady-state mean fraction $P$ of particles in the largest cluster that is reached for two initial conditions: starting from the homogeneous disordered passive suspension (solid lines, open symbols) and already containing an ordered cluster (dashed lines, closed symbols). (b) Free energy density $f(c)$ for $\sigma_{1}>0$ containing an additional $c^{4}$ term that stabilizes the high density phase. The dashed line indicates $g=g_{*}$. For $g>g_{*}$, the energy density develops a non-convex part indicating coexistence of regions of two different particle densities, which promotes hysteresis.

cally determined long-time diffusion coefficients $D(\phi)$ of the passive suspension with a quadratic function [SM]. As demonstrated in Fig. 1(a), despite these approximations we obtain excellent agreement with the numerical data.

A striking observation is made when going to lower densities $\phi_{*}<\phi \lesssim 0.32$, where clustering requires larger propulsion speeds. Here the order parameter $P$ seems to jump, which is in contrast to the continuous transition observed at higher densities. To further investigate this change, we have performed additional simulations, where we prepare a large ordered cluster consisting of $N / 2$ particles as the initial state. We let the system relax for a finite time $\left(t_{\mathrm{r}}=50\right)$ before we record the data. Fig. 2(a) shows that at larger densities indeed no hysteresis is observed, i.e., irrespective of the initial state (disordered or containing a cluster) the same steady state is reached. This is quite different for $\phi=0.3$, where a large hysteresis loop can be found. Hence, we conclude that there is a point $\left(\phi_{0} \simeq 0.32\right.$ with $\left.v_{\mathrm{c}} \simeq 66\right)$ on the instability line where the transition changes its nature from continuous to discontinuous. The continuous case is usually described as "spinodal decomposition", whereas the discontinuous behavior of the order parameter agrees with a nucleation scenario in which a sufficiently large critical nucleus has to form in order for phase separation to proceed.

Quite remarkably, this change is already contained in the mean-field description of the Cahn-Hilliard equation (see, e.g., Ref. 31]). For a qualitative insight, let us discuss the amplitude $|a|$ of "roll" perturbations $c(\mathbf{r})=$ $a e^{\mathrm{iq} \cdot \mathbf{r}}+$ c.c. $[\mathrm{SM}]$. For $\sigma_{1}<0$, the non-trivial solution for the amplitude reads

$$
|a| \sim \sqrt{-\sigma_{1} /\left(g_{*}^{2}-g^{2}\right)} .
$$

This solution exists for $g<g_{*}$ with a threshold $g_{*} \propto q$ proportional to the wave vector $q$ of the destabilizing perturbation. In this case the bifurcation is supercritical and thus indeed corresponds to a continuous growth of the amplitude as we push the system deeper into the instability region. However, when $g$ reaches $g_{*}$, this supercritical solution ceases to exist. Rather the transition becomes subcritical, i.e., there is a finite region where a stable spatially homogeneous solution $|a|=0$ and a stable solution of non-zero amplitude $|a| \neq 0$ coexist and are separated by an intermediate unstable solution. In Fig. 2(b), the bulk free energy density $f(c)$ is plotted for the different regimes, showing that for $g>g_{*}$ it becomes non-convex, which promotes a discontinuous course of the transition.

In summary, starting from the effective hydrodynamic equations obtained previously [15, we have derived an equation of motion [Eq. [11)] for the large-scale density fluctuations in a suspension of active Brownian particles close to the limit of linear stability. This evolution equation is known from the study of phase separation dynamics in passive systems as the Cahn-Hilliard equation. In particular, it implies an effective, although asymmetric, free energy without "non-integrable" terms, in spite of the genuine activity of the system. Instead of performing the double tangent construction explicitly, we have derived Eq. (13) quantifying the slope of the phase boundary. We have demonstrated excellent agreement with particle-resolved Brownian dynamics simulations. Moreover, there is a change of the transition from continuous to discontinuous, which is also in agreement with the effective theory developed here. An open question is the exponent for the scaling of the coarsening length of domains. While the Cahn-Hilliard equation for a conserved order parameter implies the exponent $1 / 3$, computer simulations of active Brownian particles in two dimensions have reported somewhat lower exponents [13, 16. However, these simulations might still be in a transient regime as is also speculated in Ref. [27]. Nevertheless, this point will have to be resolved in the future and calls for further experimental investigations.

We gratefully acknowledge support by the Deutsche Forschungsgemeinschaft through the recently established priority program 1726.

[1] S. Ramaswamy, Annu. Rev. Cond. Mat. Phys. 1, 323 (2010).

[2] M. C. Marchetti, J. F. Joanny, S. Ramaswamy, T. B. Liverpool, J. Prost, M. Rao, and R. A. Simha, Rev. Mod. Phys. 85, 1143 (2013).

[3] A. Cavagna, Phys. Rep. 476, 51 (2009). 
[4] H. H. Wensink, J. Dunkel, S. Heidenreich, K. Drescher, R. E. Goldstein, H. Löwen, and J. M. Yeomans, Proc. Natl. Acad. Sci. U.S.A. 109, 14308 (2012).

[5] V. Narayan, S. Ramaswamy, and N. Menon, Science 317, 105 (2007).

[6] J. Palacci, C. Cottin-Bizonne, C. Ybert, and L. Bocquet, Phys. Rev. Lett. 105, 088304 (2010).

[7] I. Theurkauff, C. Cottin-Bizonne, J. Palacci, C. Ybert, and L. Bocquet, Phys. Rev. Lett. 108, 268303 (2012).

[8] J. Palacci, S. Sacanna, A. P. Steinberg, D. J. Pine, and P. M. Chaikin, Science 339, 936 (2013).

[9] I. Buttinoni, J. Bialké, F. Kümmel, H. Löwen, C. Bechinger, and T. Speck, Phys. Rev. Lett. 110, 238301 (2013).

[10] M. Mijalkov and G. Volpe, Soft Matter 9, 6376 (2013).

[11] J. Palacci, S. Sacanna, A. Vatchinsky, P. M. Chaikin, and D. J. Pine, J. Am. Chem. Soc. 135, 15978 (2013).

[12] Y. Fily and M. C. Marchetti, Phys. Rev. Lett. 108, $235702(2012)$

[13] G. S. Redner, M. F. Hagan, and A. Baskaran, Phys. Rev. Lett. 110, 055701 (2013).

[14] Y. Fily, S. Henkes, and M. C. Marchetti, arXiv:1309.3714 (2013).

[15] J. Bialké, H. Löwen, and T. Speck, EPL 103, 30008 (2013).

[16] J. Stenhammar, A. Tiribocchi, R. J. Allen, D. Marenduzzo, and M. E. Cates, Phys. Rev. Lett. 111, 145702 (2013).

[17] I. Buttinoni, G. Volpe, F. Kümmel, G. Volpe, and C. Bechinger, J. Phys.: Cond. Matter 24, 284129 (2012).

[18] J. Tailleur and M. E. Cates, Phys. Rev. Lett. 100, 218103 (2008).

[19] M. E. Cates and J. Tailleur, EPL 101, 20010 (2013).

[20] R. Ni, M. A. C. Stuart, and M. Dijkstra, Nat. Commun. 4, 2704 (2013).

[21] L. Berthier, arXiv:1307.0704 (2013).

[22] J. Bialké, T. Speck, and H. Löwen, Phys. Rev. Lett. 108, 168301 (2012).

[23] A. M. Menzel and H. Löwen, Phys. Rev. Lett. 110, 055702 (2013).

[24] J. Schwarz-Linek, C. Valeriani, A. Cacciuto, M. E. Cates, D. Marenduzzo, A. N. Morozov, and W. C. K. Poon, Proc. Natl. Acad. Sci. U.S.A. 109, 4052 (2012).

[25] G. S. Redner, A. Baskaran, and M. F. Hagan, Phys. Rev. E 88, 012305 (2013).

[26] B. M. Mognetti, A. Šarić, S. Angioletti-Uberti, A. Cacciuto, C. Valeriani, and D. Frenkel, Phys. Rev. Lett. 111, 245702 (2013)

[27] R. Wittkowski, A. Tiribocchi, J. Stenhammar, R. J. Allen, D. Marenduzzo, and M. E. Cates, arXiv:1311.1256 (2013).

[28] M. E. Cates, D. Marenduzzo, I. Pagonabarraga, and J. Tailleur, Proc. Natl. Acad. Sci. U.S.A. 107, 11715 (2010).

[29] M. C. Cross and P. C. Hohenberg, Rev. Mod. Phys. 65, 851 (1993).

[30] J. W. Cahn and J. E. Hilliard, J. Chem. Phys. 28, 258 (1958).

[31] A. Novick-Cohen, J. Stat. P 38, 707 (1985).

\section{STRENGTH OF THE NON-LINEAR TERM}

Here we give the explicit expression for $g=2 \xi_{\mathrm{c}}\left(\alpha_{0}+\right.$ $\left.\beta_{0}\right)$. In the main text, we have defined

$$
\xi \equiv \frac{\bar{\rho} \zeta}{v_{*}}, \quad \alpha \equiv 4\left(v_{0} / v_{*}-\xi\right), \quad \beta \equiv 2\left(v_{0} / v_{*}-2 \xi\right) .
$$

Moreover, at the linear stability limit, we have

$$
\xi_{\mathrm{c}}=\frac{3}{4} u-\frac{1}{4} \sqrt{u^{2}-1}
$$

with dimensionless critical speed $u \equiv v_{\mathrm{c}} / v_{*}$. At this critical speed

$$
\alpha_{0}=4\left(u-\xi_{\mathrm{c}}\right), \quad \beta_{0}=2\left(u-2 \xi_{\mathrm{c}}\right) .
$$

We can thus express the non-linear coefficient $g$ in terms of $u$,

$$
\begin{aligned}
g(u) & =2 \xi_{\mathrm{c}}\left(\alpha_{0}+\beta_{0}\right)=4 \xi_{\mathrm{c}}\left(3 u-4 \xi_{\mathrm{c}}\right) \\
& =1-u^{2}+3 u \sqrt{u^{2}-1} \\
& \geqslant 0,
\end{aligned}
$$

where the equal sign holds for $u=1$.

\section{MINIMAL VELOCITY}

The minimal critical velocity for the dynamical phase separation to be possible is $v_{*}=4 \sqrt{D D_{\mathrm{r}}}$, where we estimate $D$ from the long-time passive diffusion coefficient. The numerically determined diffusion coefficients are well fitted by the quadratic function

$$
D(\phi) \simeq-0.76 \phi^{2}-0.34 \phi+0.72,
$$

see Fig. 3

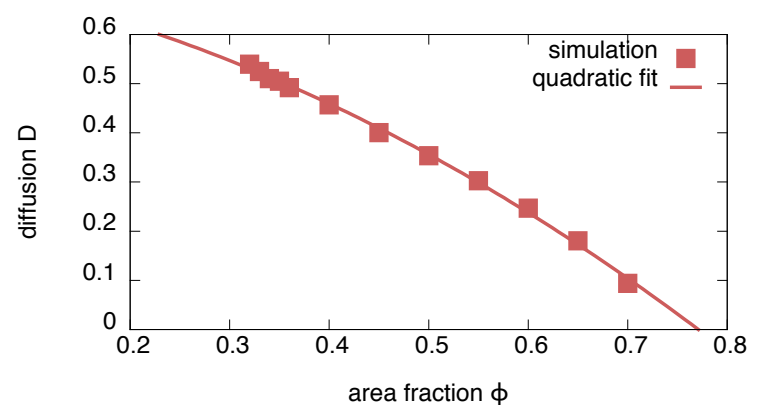

FIG. 3: Long-time diffusion coefficients of the passive $\left(v_{0}=0\right)$ suspension as a function of the area fraction $\phi$.

\section{AMPLITUDE EQUATION}

For completeness, we present the derivation of the amplitude equation for roll solutions following standard arguments, see Ref. 29] for a comprehensive review. Specifically, we follow the route described in Ref. 31. Since 

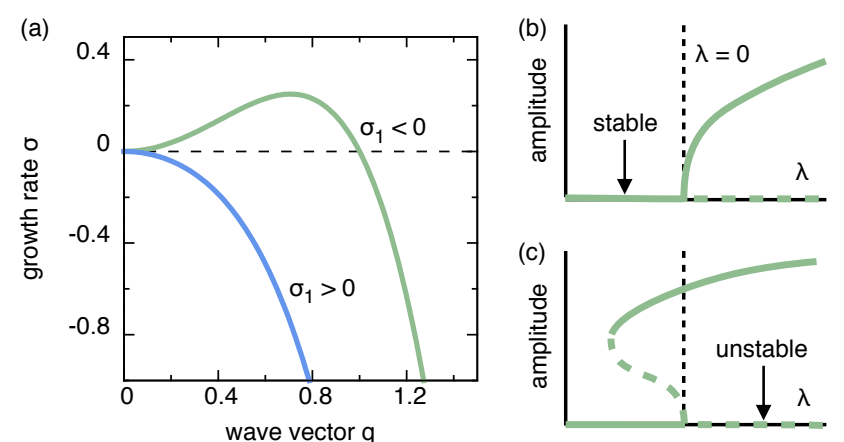

FIG. 4: (a) Growth rate as a function of the wave vector $q$ for the linearly unstable $\left(\sigma_{1}<0\right)$ and linearly stable $\left(\sigma_{1}>0\right)$ regime. (b) Bifurcation diagram for the supercritical (continuous transition) and (c) the subcritical (discontinuous transition) case.

we are mainly interested in the qualitative behavior, we simply augment the effective free energy with a term proportional to $c^{4}$ in order to take into account the volume exclusion. The Cahn-Hilliard equation then reads

$$
\partial_{t} c=\nabla^{2}\left[\sigma_{1} c-g c^{2}+\kappa c^{3}-\nabla^{2} c\right] .
$$

From this equation we obtain the linear dispersion relation $\hat{\sigma}(q)=-\sigma_{1} q^{2}-q^{4}$, which reaches its maximum at $q_{\mathrm{c}}^{2}=-\sigma_{1} / 2$ and becomes zero for $q_{0}^{2}=-\sigma_{1}$ in the case of $\sigma_{1}<0$, see Fig. 4(a). The wave vector $q_{0}$ marks the boundary of linear stability, i.e. for wave vectors $q>q_{0}$, the unperturbed solution is still linearly stable.

We aim to investigate periodic structures with a wave vector $q$ close to $q_{0}, q^{2}=q_{0}^{2}-\lambda \epsilon^{2}$, where $\lambda$ corresponds to the "quench depth". The growth rate is $\hat{\sigma}(q) \sim \lambda q_{0}^{2} \epsilon^{2}$. This suggests to introduce an even slower time scale $s=$ $\epsilon^{2} t$ leading to

$$
\epsilon^{2} \partial_{s} c=L c+\nabla^{2}\left[-\lambda \epsilon^{2} c-g c^{2}+\kappa c^{3}\right]
$$

with the linear self-adjoint operator $L=-q^{2} \nabla^{2}-\nabla^{4}$ and $\sigma_{1}=-q^{2}-\lambda \epsilon^{2}$. The next step is to expand $c(\mathbf{r}, s)=$ $\epsilon c_{1}(\mathbf{r}, s)+\epsilon^{2} c_{2}(\mathbf{r}, s)+\cdots$ into powers of $\epsilon$.

Specifically, we are interested in periodic "roll" solutions of the form

$$
c_{1}(\mathbf{r}, s)=a(s) e^{\mathrm{iq} \cdot \mathbf{r}}+\text { c.c. }
$$

with complex amplitude $a$. To linear order, we find $L c_{1}=$ 0 . Hence, the amplitude of the linear solution would grow in an unbounded way. However, the non-linear terms in Eq. (21) couple this solution to higher wave-vectors and thus lead to a saturation of the amplitude. To second order, we obtain

$$
0=L c_{2}-g \nabla^{2} c_{1}^{2}
$$

We make the ansatz

$$
c_{2}(\mathbf{r}, s)=b(s) e^{2 \mathbf{i q} \cdot \mathbf{r}}+\text { c.c. }
$$

leading to

$$
0=-12 q^{4} b+4 g q^{2} a^{2}, \quad b=\frac{g}{3 q^{2}} a^{2} .
$$

To order $\epsilon^{3}$, we obtain

$$
\partial_{s} c_{1}=L c_{3}+\nabla^{2}\left[-\lambda c_{1}-2 g\left(c_{1} c_{2}\right)+\kappa c_{1}^{3}\right]
$$

We multiply this equation by $e^{-\mathrm{iq} \cdot \mathbf{r}}$ and integrate over an area $A$, where the edge length in the direction of $\mathbf{q}$ is set to a multiple of $2 \pi / q$. Only terms that have a spatial dependence $e^{\mathrm{iq} \cdot \mathbf{r}}$ will survive the integration. Hence, the amplitude equation becomes

$$
\begin{aligned}
\dot{a} & =q^{2} \lambda a+2 g q^{2} a^{*} b-3 \kappa q^{2} a^{*} a^{2} \\
& =q^{2} \lambda a+\frac{2}{3}\left(g^{2}-\frac{9}{2} \kappa q^{2}\right)|a|^{2} a
\end{aligned}
$$

where $a^{*}$ denotes the complex conjugate of $a$ and $|a|^{2}=$ $a a^{*}$. The stationary solutions $(\dot{a}=0)$ are $|a|=0$ and

$$
|a|^{2}=\frac{\frac{3}{2} q^{2} \lambda}{\frac{9}{2} \kappa q^{2}-g^{2}}=\frac{\lambda}{3 \kappa} \frac{1}{1-\left(g / g_{*}\right)^{2}}
$$

with $g_{*}^{2}=\frac{9}{2} \kappa q^{2}$.

We can distinguish between the two cases $g<g_{*}$ and $g>g_{*}$ : (i) For $g<g_{*}$ and $\lambda<0$ we only find the trivial solution $a=0$. Increasing $\lambda$ to $\lambda>0$, the trivial solution becomes unstable and we obtain the amplitude $|a| \sim \sqrt{\lambda}$. This behavior marks a supercritical bifurcation corresponding to a continuous transition. (ii) On the contrary, for $g>g_{*}$ only the trivial solution $|a|=0$ follows for $\lambda>0$, and it is unstable. However, two solutions are obtained from the above procedure for $\lambda<0$ : $|a|=0$, which is now stable, and an unstable solution $|a| \sim \sqrt{-\lambda}$. Hence, at the threshold $g=g_{*}$, the nature of the bifurcation changes from supercritical to subcritical. In Fig. 4(b) and (c), corresponding bifurcation diagrams are sketched.

Finally, we rescale $c=\left(3\left|\sigma_{1}\right| / g_{*}\right) \hat{c}$ and eliminate $\kappa$ in favor of $g_{*}$. We test for wave vectors of magnitude $q^{2}=$ $\left|\sigma_{1}\right| / 2$, which corresponds to $q=q_{\mathrm{c}}$ of the fastest growing perturbation in the case of $\sigma_{1}<0$. This implies $\lambda=$ $-\sigma_{1} / 2$, and we can write the bulk free energy density as

$$
f(\hat{c})=\frac{9\left|\sigma_{1}\right|^{3}}{g_{*}^{2}}\left\{ \pm \frac{1}{2} \hat{c}^{2}-\frac{g}{g_{*}} \hat{c}^{3}+\hat{c}^{4}\right\}
$$

for $\sigma_{1}>0$ and $\sigma_{1}<0$, respectively. This function is plotted in Fig. 2(b) in the main text. In agreement with the analysis of the amplitude equation, the bulk free energy becomes non-convex for $g>g_{*}$, in line with a subcritical bifurcation. 